\title{
Predicting Axonal Response to Molecular Gradients with a Computational Model of Filopodial Dynamics
}

\author{
Geoffrey J. Goodhill \\ geoff@georgetown.edu \\ Department of Neuroscience, Georgetown University Medical Center, \\ Washington, D.C. 20007, U.S.A. \\ Ming Gu \\ gu@klab.caltech.edu \\ Computation and Neural Systems, California Institute of Technology \\ Pasadena, CA 91125, USA
}

Jeffrey S. Urbach

urbach@physics.georgetown.edu

Department of Physics, Georgetown University,

Washington, D.C. 20057, U.S.A.

Axons are often guided to their targets in the developing nervous system by attractive or repulsive molecular concentration gradients. We propose a computational model for gradient sensing and directed movement of the growth cone mediated by filopodia. We show that relatively simple mechanisms are sufficient to generate realistic trajectories for both the short-term response of axons to steep gradients and the long-term response of axons to shallow gradients. The model makes testable predictions for axonal response to attractive and repulsive gradients of different concentrations and steepness, the size of the intracellular amplification of the gradient signal, and the differences in intracellular signaling required for repulsive versus attractive turning.

\section{Introduction}

A key method used by developing axons to navigate to appropriate targets in the embryonic nervous system is guidance by molecular gradients (Mueller, 1999; Song \& Poo, 2001; Yu \& Bargmann, 2001; Dickson, 2002, Huber, Kolodkin, Ginty, \& Cloutier 2003; Guan \& Rao, 2003). Both gradient sensing and directed movement are primarily mediated by the growth cone, a highly specialized structure at the axonal tip (Gordon-Weeks, 2000). Growth cones typically consist of a central zone surrounded by web-like veils, the lamellipodia, and highly dynamic spike-like protrusions, the filopodia, which usually have an average lifetime of just a few minutes (Rehder 
\& Kater, 1996). Growth cones can display turning responses in a few minutes when exposed to a steep gradient in a two-dimensional culture system (e.g. Gundersen \& Barrett, 1979; Zheng, Felder, Conner, \& Poo, 1994; Zheng, Wan, \& Poo, 1996; Ming et al., 1997; Song, Ming, \& Poo, 1997; de la Torre, Höpker, Ming, \& Poo, 1997; Song et al., 1998; Hong, Hinck, Nishiyama, Poo, \& Tessier-Lavigne, 1999; Hong, Nishiyama, Henley, Tessier-Lavigna, \& Poo, 2000; Zheng, 2000; Ming et al., 2002; Nishiyama et al., 2003). When exposed to a shallow gradient generated by diffusion from nearby tissue in a three-dimensional culture, system growth cones display quite variable trajectories, but with a tendency for smooth turning up (or down for repulsive factors) the gradient on a timescale of several hours (e.g. Lumsden \& Davies, 1983, 1986; Tessier-Lavigne, Placzek, Lumsden, Dodd, \& Jessell, 1988; Colamarino \& Tessier-Lavigne, 1995; Keynes et al., 1997; Richards, Koester, Tuttle, \& O'Leary, 1997; Varela-Echavarria, Tucker, Püschel, \& Guthrie, 1997; Brose et al., 1999; Braisted et al., 2000; Caton et al., 2000; Patel et al., 2001; Nguyen Ba-Charvet et al., 2001; Shu \& Richards, 2001; Anderson et al., 2003, Charron, Stein, Jeong, McMahon, \& Tessier-Lavigne, 2003). Attraction can sometimes be converted to repulsion, and vice versa, by methods including manipulation of the levels of cyclic nucleotides in the growth cone (Song et al., 1997, 1998; Song \& Poo, 2001; Nishiyama et al., 2003), by growing on alternative substrates (Höpker, Shewan, Tessier-Lavigne, Poo, \& Holt, 1999), and electrical activity (Ming, Henley, Tessier-Lavigne, Song, \& Poo, 2001). The axonal trajectories generated by repulsion roughly mirror those generated by attraction. Several lines of evidence suggest that filopodia often make a critical contribution to the sensing and movement capabilities of the growth cone both in vitro and in vivo (Argiro, Bunge, \& Johnson, 1985; O'Connor, Duerr, \& Bentley, 1990; Myers \& Bastiani, 1993; Davenport, Dou, Rehder, \& Kater, 1993; Zheng et al., 1996; Steketee \& Tosney, 1999; but see also Wang, Liu, Diefenbach, \& Jay, 2003). When the filopodia are eliminated, growth cones cannot navigate their environment and do not respond to either substrate bound or diffusible guidance cues (Bentley \& Toroian-Raymond, 1986; Chien, Rosenthal, Harris, \& Holt, 1993). In a steep gradient, in vitro filopodia become asymmetrically distributed toward the source of an attractive factor and away from the source of a repulsive factor on the timescale of a few minutes (Zheng et al., 1996; Zheng, 2000).

The general issue of chemotaxis by small sensing devices has been extensively studied experimentally in systems such as bacteria, leukocytes, and slime molds (e.g., Devreotes \& Zigmond, 1988; Eisenbach, 1996; Parent \& Devreotes, 1999). In addition, chemotaxis in these systems has been subjected to a variety of theoretical analyses (Tranquillo, 1990), including predictions of the minimum detectable gradient steepness (Berg \& Purcell, 1977), models of the trajectories generated (Tranquillo \& Lauffenburger, 1987), and models of the intracellular transduction pathways that mediate response to gradients (Moghe \& Tranquillo, 1995; Barkai \& Leibler, 1997). However, such quantitative analyses are much less well developed for growth cones 
(Buettner, 1995; Robert \& Sweeney, 1997; Hely \& Willshaw, 1998; Goodhill, 1998; Goodhill \& Urbach, 1999; Meinhardt, 1999). In particular, the theoretical consequences for growth cone gradient sensing of the unique dynamical properties of filopodia remain unexplored, and the precise requirements for generating similar trajectories to those seen experimentally in both attractive and repulsive gradients have not been investigated.

Here, we present the first quantitative model for how filopodia mediate both attractive and repulsive gradient-sensing and -directed movement by growth cones. Our basic hypothesis is that new filopodia tend to be generated in the direction where ligand binding is highest, in the case of attraction, and lowest, in the case of repulsion, and that the growth cone takes a turn toward the average direction of the filopodia. We show that this hypothesis is sufficient to account for the trajectories generated by growth cones in both attractive and repulsive gradients on both short and long timescales. The model is expressed at the level of filopodial dynamics and does not address in detail the sequence of intracellular signaling events leading from receptor binding to the spatially anisotropic polymerization and bundling of actin that actually causes filopodial extension (Song \& Poo, 2001; Tanaka \& Sabry, 1995; Suter \& Forscher, 2000; Steketee, Balazovich, \& Tosney, 2001; Zhou, Waterman-Storer, \& Cohan, 2002). However, the model makes a number of predictions about the mathematical constraints that these intracellular transformations should satisfy to reproduce trajectories seen experimentally. In particular, it predicts the degree of amplification required from the binding signal to the turning signal and how the signal transduction pathways mediating attraction may differ from those mediating repulsion. The model also suggests that the response to attractive and repulsive gradients may not be entirely symmetric.

\section{The Model}

We consider an idealized growth cone consisting of a two-dimensional semicircular body from which several one-dimensional filopodia extend (see Figure 1A). Both the (one-dimensional) surface of the growth cone body and the filopodia are covered with receptors at random locations. The probability $p_{i}$ for receptor $i$ to be bound is given by $p_{i}=C_{i} /\left(C_{i}+K_{D}\right)$, where $C_{i}$ is the external ligand concentration at the position of receptor $i$ and $K_{D}$ is the dissociation constant for the receptor-ligand complex. At each time step, the state of each receptor (bound or unbound) is updated by random assignment with this probability. Receptor binding is averaged within a local region (hereafter referred to as a bin), with bins equally spaced around the growth cone. Receptor binding is then divided by the total number of receptors in that bin to give the fraction of bound receptors per bin, $b(\theta)$. This binding signal is assumed to establish a shallow internal gradient of an intracellular signaling molecule. This shallow gradient by itself is insufficient to generate the degree of turning shown by real axons in gradients (see 
A

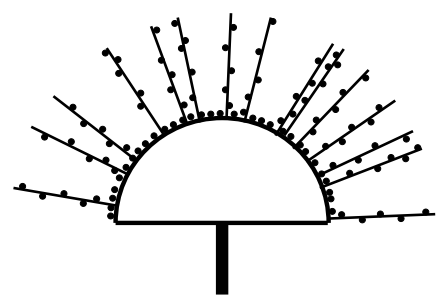

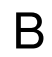

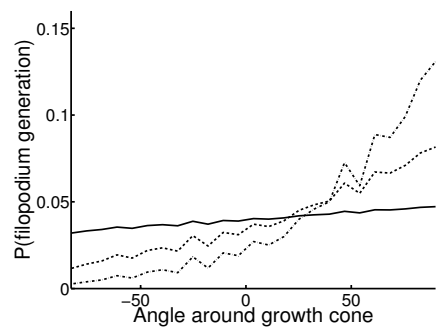

Figure 1: The model. (A) Model growth cone. The small circles represent receptors, which are distributed randomly on the surface of both the growth cone and the filopodia. (B) Schematic example of the internal signaling gradient generated after amplification. An attractive external ligand gradient points to the right of the growth cone, and the concentration at the center of the growth cone is $K_{D}$. Solid line: no amplification (i.e., $b(\theta)$ ). Dashed line: amplification $=5$ (i.e., $b^{5}(\theta)$ ). Dash-dotted line: amplification $=10$ (i.e., $b^{10}(\theta)$ ). Note that the larger the amplification, the higher the probability is that a new filopodium will be generated on the right rather than the left of the growth cone (though there is always a nonzero probability of a filopodium appearing at any angle). For clarity, a fractional change in concentration of $50 \%$ across the growth cone is shown; the gradients actually used in the model are much shallower than this.

section 3). We therefore further assume that there is an amplification step between this gradient and the probability of generating a new filopodium. In reality, this amplification arises from a complex network of intracellular signalling (Parent \& Devreotes, 1999; Song \& Poo, 2001; Guan \& Rao, 2003). The precise details of this network are not yet well understood for growth cones; we simply assume that its ultimate effect is to raise the shallow internal gradient to a power $n$ (see Figure 1B; this is somewhat analogous to the standard assumption in many neural network models that the output of a neuron is simply a sigmoid function of its input (Haykin, 1999)). This amplified gradient then gives the probability $P(\theta)$ of generating a new filopodium in each bin, that is, $P(\theta) \propto b^{n}(\theta)$ in the attractive case. For repulsive ligand gradients, an inversion step is required. We consider three forms: $P(\theta) \propto(1-b(\theta))^{n}, P(\theta) \propto 1-b^{n}(\theta)$, and $P(\theta) \propto b^{-n}$ (see section 5). The bin from which a new filopodium will extend is then chosen from this probability distribution, and a new filopodium is extended from a position chosen from a uniform distribution within this bin. At the same time, the oldest filopodium is retracted, maintaining a constant number of filopodia on the growth cone. The growth cone then moves a constant small distance, mostly in the forward direction but with a slight deviation to one side or the other (a rate of growth that depends on the concentration of factor could 
be added as appropriate). This deviation is in the direction defined by the net pull of the filopodia. The entire cycle of receptor binding, internal gradient establishment, amplification, bin selection, filopodia generation, and directed movement is then repeated. We have investigated two versions of the model. In the angle version, the distance the growth cone moves forward at each time step is constant, and the deviation is toward the mean angle of the filopodia. In the force version, the distance moved and deviation are proportional to the force exerted by the filopodia, assuming each pulls equally along its length. The results generated by these two versions of the model are very similar, and we therefore present results only for the simpler angle model (see also section 4 ).

2.1 Parameters. Unless otherwise stated, the parameter values used to generate the data shown in section 3 were those listed below and in Table 1, which also lists some typical values from the experimental literature. This list represents a small fraction of the quantitative data in the literature, and in some axon guidance contexts, different parameter values would be appropriate. Additional parameter values were as follows. The number of receptors on the growth cone was 3000 in total, with 100 each per filopodium (1500 total on filopodia) and the remaining 1500 distributed on the body of

Table 1: Parameters Used in the Model.

\begin{tabular}{|c|c|c|c|c|c|}
\hline Parameter & $\begin{array}{l}\text { Model } \\
\text { Value }\end{array}$ & $\begin{array}{l}\text { Experimental } \\
\text { Values }\end{array}$ & Axon Type & Substrate & References \\
\hline $\begin{array}{l}\text { Growth cone } \\
\text { width (microns) }\end{array}$ & 20 & $\begin{array}{l}18 \\
10\end{array}$ & $\begin{array}{l}\text { DRG } \\
\text { Xenopus spinal }\end{array}$ & $\begin{array}{l}\text { Glass, PLL } \\
\text { Glass }\end{array}$ & $\begin{array}{l}\text { Bray \& Chapman, } 1985 \\
\text { Zheng et al., } 1996\end{array}$ \\
\hline $\begin{array}{l}\text { Growth cone } \\
\text { speed } \\
\text { (microns/hour) }\end{array}$ & 20 & $\begin{array}{l}20 \pm 2,18 \pm 4 \\
18-24 \\
35-45 \\
11-18 \text { (turning) } \\
40 \text { (not turning) }\end{array}$ & $\begin{array}{l}\text { PC12, SCG } \\
\text { Xenopus spinal } \\
\text { Zebrafish retinal } \\
\text { Xenopus spinal }\end{array}$ & $\begin{array}{l}\text { Collagen } \\
\text { Glass } \\
\text { In vivo } \\
\text { Glass }\end{array}$ & $\begin{array}{l}\text { Aletta \& Greene, } 1988 \\
\text { Zheng, 2000 } \\
\text { Hutson \& Chien, } 2002 \\
\text { Zheng et al., } 1996\end{array}$ \\
\hline $\begin{array}{l}\text { Number of } \\
\text { filopodia per }\end{array}$ & 15 & $\begin{array}{l}4-6 \\
6\end{array}$ & $\begin{array}{l}\text { Zebrafish retinal } \\
\text { LBD, SND }\end{array}$ & $\begin{array}{l}\text { In vivo } \\
\text { In vivo }\end{array}$ & $\begin{array}{l}\text { Hutson \& Chien, } 2002 \\
\text { Kim, Kolodziej, \& } \\
\text { Chiba, } 2002\end{array}$ \\
\hline growth cone & & $8-20$ & Xenopus spinal & Glass & Zheng et al., 1996 \\
\hline Filopodium & 10 & $10-25$ & PC12, SCG & Collagen & Aletta \& Greene, 1988 \\
\hline \multirow[t]{4}{*}{ length (microns) } & & $\begin{array}{l}6.9 \pm 0.4 \\
5-15\end{array}$ & $\begin{array}{l}\text { DRG } \\
\text { Aplysia }\end{array}$ & $\begin{array}{l}\text { Glass, PLL } \\
\text { PLL }\end{array}$ & $\begin{array}{l}\text { Bray \& Chapman, } 1985 \\
\text { Goldberg \& } \\
\text { Burmeister, } 1986\end{array}$ \\
\hline & & $3-4$ & Zebrafish retinal & in vivo & Hutson \& Chien, 2002 \\
\hline & & $4-7$ & LBD, SND & in vivo & $\begin{array}{l}\text { Kim, Kolodziej, \& } \\
\text { Chiba, } 2002\end{array}$ \\
\hline & & 9 & Xenopus spinal & Glass & Zheng et al., 1996 \\
\hline Filopodium & 7.5 & 6 & LBD, SND & & Kim et al., 2002 \\
\hline lifetime (min) & & 7.5 & Xenopus spinal & Glass & $\begin{array}{l}\text { Gomez, Robles, Poo, } \\
\& \text { Spitzer, } 2001\end{array}$ \\
\hline
\end{tabular}

Notes: Parameter values used in the simulations reported here and some representative values from the literature. Note that since the number of filopodia is assumed fixed, the filopodium lifetime determines the size of each time step: for the parameters here, the time step is 30 seconds. DRG, dorsal root ganglion; PLL, poly-L lysine; SCG, superior cervical ganglion. 
the growth cone. The number of angle bins was 25 (an average of 120 receptors per bin). The ligand concentration was equal to $K_{D}$ at the starting point of each growth cone trajectory. The concentrations of diffusible attractants encountered by axons in vivo are generally unknown, but $K_{D}$ is the concentration at which growth cones are expected to be maximally sensitive to gradients (Tranquillo, 1990). The gradient was exponential, with a steepness (expressed as percentage change in ligand concentration across $10 \mu \mathrm{m}$ ) varying from $0.1 \%$ to $10 \%$. The new orientation of the growth cone is equal to 0.97 times the previous orientation, plus 0.03 times the average angle of the filopodia. This value controls how quickly the axon reorients in response to changes in filopodia distribution. Values much lower than 0.97 produced trajectories for long-term responses that were more convoluted than those observed experimentally. The length of each time step was 30 seconds, and the distance moved forward in this time was $1 / 6 \mu \mathrm{m}$ (equivalent to $20 \mu \mathrm{m} /$ hour). At successive time steps, the growth cone makes a statistically independent measurement of the concentration in each bin. For diffusion-limited receptor-binding kinetics, the minimum time between statistically independent concentration measurements can be estimated to be the radius of the sensing device squared divided by the diffusion constant (Berg \& Purcell, 1977). For diffusion constants in the range $10^{-6}$ to $10^{-7}$ $\mathrm{cm}^{2} / \mathrm{sec}$, this time is between 1 and 10 seconds, which as required is less than the time step used in the model. Each individual axon was initialized with a different random seed and a different initial distribution of filopodia. The total time simulated was 2 hours for "short" trajectories and 40 hours for "long" trajectories.

\section{Results}

Our model represents the main steps that may occur inside the growth cone to convert a shallow external ligand gradient to directed movement (see Figure 1). The gradient of receptor binding over the surface of the growth cone produces a difference in the likelihood that filopodia will be generated on one side of the growth cone compared to the other. This difference could arise, for example, from an internal gradient in actin polymerization or bundling triggered by a gradient of an intracellular signaling molecule. In each time step, the growth cone moves forward and turns slightly toward the net direction of the filopodia, due either to the forces generated by filopodial adhesions or the asymmetric effects of the filopodia on microtubule dynamics. Two crucial components in the conversion from the initial shallow gradient to the gradient of probability in filopodium generation are amplification and inversion. Amplification is the process that steepens the initial gradient so that it is effective for guidance (illustrated in Figure 1B). Inversion is required for repulsive ligand gradients: besides being amplified, the direction of the initial shallow gradient must now be reversed so 
that filopodia are more likely to be generated on the down-gradient side of the growth cone than the up-gradient side.

Growth cones can display a wide variety of behaviors in different contexts. For instance, a single filopodial contact can sometimes cause a complete redirection of the growth cone ( $\mathrm{O}^{\prime}$ Connor et al., 1990). Our concern was to model two specific well-characterized behaviors of axons growing in or on a uniform substrate: a rapid turn in response to a steep external ligand gradient and a slow turn in response to a shallow external ligand gradient. Experimental data for the first case come primarily from Poo and colleagues (Zheng et al., 1994, 1996; Ming et al., 1997, 2002; de la Torre et al., 1997; Song et al., 1997, 1998; Hong et al., 1999, 2000; Zheng, 2000; Nishiyama et al, 2003). By slowly ejecting a chemotropic factor from a pipette near to a growth cone growing on a two-dimensional substrate covered in fluid medium, gradients are established of steepness $5 \%$ to $10 \%$ over $10 \mu \mathrm{m}$ at the growth cone (Zheng et al., 1994). Under appropriate conditions, the axons turn toward (or away from) the pipette on a timescale of the order of 1 hour. Experimental data regarding the long-term response of axons to shallow gradients come from the 3D collagen gel assay (e.g., Lumsden \& Davies, 1983, 1986; Tessier-Lavigne et al., 1988; Colamarino \& Tessier-Lavigne, 1995; Keynes et al., 1997; Richards et al., 1997; Varela-Echavarria et al., 1997; Brose et al., 1999; Braisted et al., 2000; Caton et al., 2000; Patel et al., 2001; Nguyen Ba-Charvet et al., 2001; Shu \& Richards, 2001; Anderson et al., 2003; Charron et al., 2003). Here, a target explant of factor-secreting tissue or a block of, for example, 293T cells transfected with the factor is placed close to an explant containing the neurons under investigation. Under appropriate conditions, axons emerge from the latter explant and grow preferentially toward (or away from) the target on a timescale of the order of 1 day. This is generally interpreted to mean that the factor has diffused from the target into the collagen, creating a gradient (Goodhill, 1997, 1998), though such gradients have not been directly measured.

To investigate the behavior of the model for short trajectories in a steep attractive gradient, we simulated 100 axons, each starting from the origin and initially moving in the positive y direction (see Figure 2). An exponential gradient with a fractional change of either $0 \%$ or $5 \%$ per $10 \mu \mathrm{m}$ was present directed along the $x$-axis, at an angle of 90 degrees to the initial trajectory. Parameter values are given in section 2, and the relationship between filopodial initiation probability $P$ and receptor binding $b$ was given by $P \propto b^{n}$. Figures $2 \mathrm{~A}$ and $2 \mathrm{~B}$ show a typical set of trajectories for an amplification factor $\mathrm{n}=10$ for zero gradient and a $5 \%$ gradient, respectively (Zheng et al., 1994, 1996; Ming et al., 1997, 2002; de la Torre et al., 1997; Song et al., 1997, 1998; Hong et al., 1999, 2000; Zheng, 2000) Analysis of the model below shows that any combination of amplification and steepness with the same product (e.g., $n=5$, steepness $=10 \%$ ) would produce approximately the same effect. Figure $2 \mathrm{C}$ shows the cumulative distribution of final turning angles for zero gradient, amplification $=10$, and for a $5 \%$ 
A

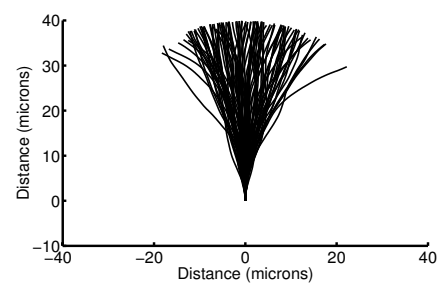

C

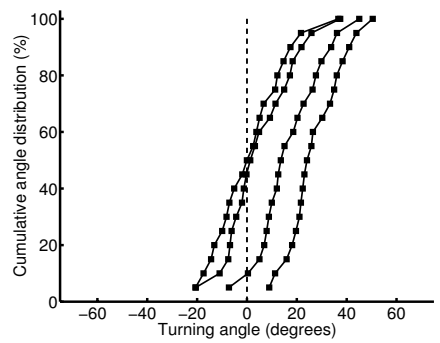

B

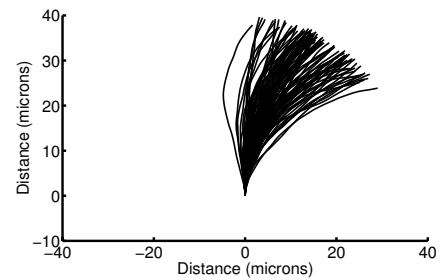

D

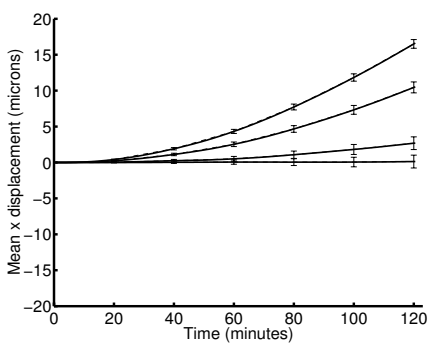

Figure 2: Short axon trajectories generated by the model. An attractive gradient of steepness $0 \%$ (A) or $5 \%$ (B) points to the right. One hundred simulated axons are shown, each starting from a different random seed. Amplification $=10$ in both cases. Growth cones themselves are not drawn. (C) Cumulative distribution of final axon turning angles. The left-most curve is for zero gradient; the other three curves moving from left to right are for a $5 \%$ gradient with amplification $=1,5,10$. (D) Mean $x$ displacement of each population of 100 axons as a function of time. The four curves from bottom to top correspond to the four curves from left to right in $\mathrm{C}$.

gradient, amplification $=1,5,10$. An alternative measure of the response to the cumulative distribution is the mean distance each population of axons has moved in the $x$ direction as a function of time (see Figure 2D). For amplification $=1$, only a modest degree of turning up the gradient is seen, and there is a wide spread in trajectories, while for amplification $=10$, there is more robust turning and a smaller spread of trajectories. It is thus clear that amplification in the range 5 to 10 in the model is necessary to reproduce the behavior of axons seen experimentally. We also investigated the sensitivity of the model to the number of filopodia used in the range 5 to 25 (data not shown); there were no significant changes in the observed behavior. The model is somewhat sensitive to the total number of receptors on the growth cone. The mean $x$ displacement after 2 hours increased slightly as recep- 
A

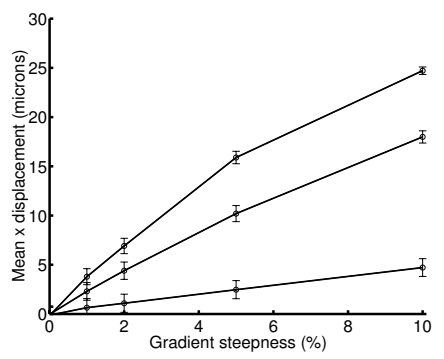

C

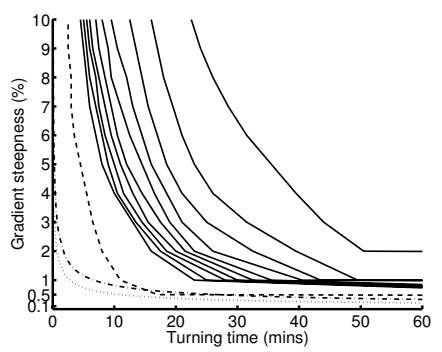

B

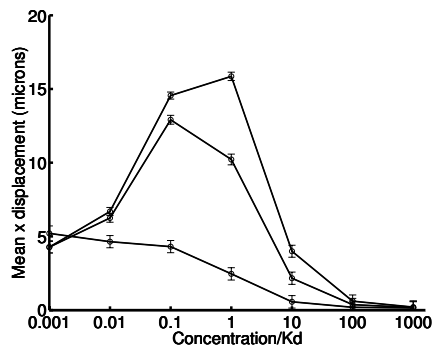

Figure 3: Statistics of short axon trajectories. (A) Mean $\times$ displacement after 2 hours as a function of gradient steepness. Error bars are standard error in the mean (SEMs) averaged over 100 axons per run. The three curves are amplification $=1,5,10$ from bottom to top. (B) Mean $x$ displacement as a function of concentration at the growth cone, for amplification $=1,5,10$. Error bars are SEMs averaged over five runs of 100 axons per run. (C) Gradient steepness versus turning time. Solid lines are amplification $=1$ to 10 moving from right to left. Dashed line: amplification $=100$. Dotted line: theoretical prediction based on thermal noise from Berg and Purcell (1977). Dot-dash line: theoretical prediction based on receptor noise from Tranquillo (1990).

tor numbers increased above 3000 and decreased significantly as receptor numbers decreased below 1000 (data not shown).

The model produces specific predictions for how the axonal response to gradients varies with gradient steepness and with absolute concentration (Goodhill, 1998; Goodhill \& Urbach, 1999). Figure 3A plots the mean $x$ displacement after 2 hours as a function of (attractive) gradient steepness for amplification $=1,5,10$. As expected, the mean $x$ displacement increases with increasing gradient steepness. Figure 3B plots $x$ displacement as a function of the concentration at the starting position of the growth cones for amplification $=1,5,10$. It can be seen that the response drops off 
away from $C=K_{d}$. For high concentrations, most receptors are bound most of the time, whereas for low concentrations, hardly any receptors are bound, and in neither case is it possible to detect a small difference in average binding between the two sides of the growth cone. This roughly matches data for leukocyte chemotaxis (Zigmond, 1977), and for growth cones using a new experimental assay we have recently developed (Rosoff et al., 2004).

In order to compare the behavior of the model with theoretical predictions regarding gradient detection by small sensing devices, we simulated gradient steepness versus turning time, defined as the first time at which the mean $x$ displacement exceeds the standard deviation of $x$ displacement (see Figure 3C). As expected, the greater the amplification, the shorter is the turning time for a given gradient steepness. Also shown in Figure 3C is the theoretical prediction of Berg and Purcell (1977), as applied to growth cones (Goodhill \& Urbach, 1999). This model calculates the unavoidable thermal fluctuations in the local concentration of chemoattractant, and thus the minimum possible detectable gradient steepness. Here, turning time is taken to be the time over which the sensing device averages concentration measurements before making a decision about the direction of the gradient. There are at least three effects that reduce the senstivity of our model below the theoretical maximum. One effect is the randomness arising from the generation of new filopodia (see section 2), which is included to model intracellular noise. This effect can be effectively elimated by using an extremely high value of the amplification, such as that used to generate the dashed line (amplification =100). A second effect is the noise arising from the inherently stochastic receptor binding. Another curve in Figure 3C shows the maximum possible turning time after allowing for receptor noise (Tranquillo, 1990). Finally, at short times, the turning time is limited by the fact that the growth cone can reorient a relatively small amount in each time step (see section 2; without this inertia, the trajectories are highly irregular and do not match those seen in experiments).

Besides being attracted by molecular gradients, axons can often also be repelled by these gradients (Song et al., 1997, 1998; Song \& Poo, 2001; Nishiyama et al., 2003). We used the same model parameters for repulsion as for attraction, except that now filopodia are preferentially generated on the side of the growth cone facing down the gradient. Figure $4 \mathrm{~A}$ shows repulsive turning when the the probability of generating a new filopodium is given by $P(\theta) \propto(1-b(\theta))^{n}(5 \%$ gradient to the right, amplification $=$ 10). Figure $4 \mathrm{~B}$ shows response as a function of concentration. This curve is similar to that for attractive turning shown in Figure 3B, except reversed: in the repulsive case, sensitivity drops off faster at low concentration than high concentration, whereas the opposite is true for attraction. We also investigated $P(\theta) \propto b^{-n}$ and $P(\theta) \propto\left(1-b^{n}(\theta)\right)$ (data not shown). The former case produced a response as a function of concentration very similar to the attractive case, while the latter case produced very little turning. The bio- 
A

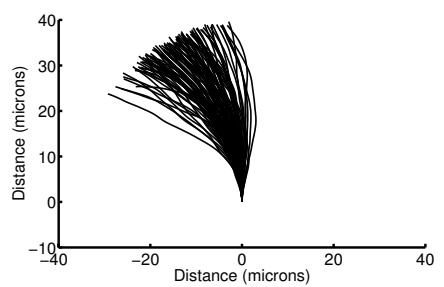

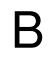

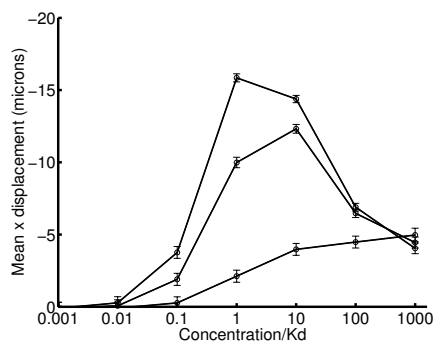

Figure 4: Short trajectories in a repulsive gradient. (A) As before, the gradient increases to the right (steepness $=5 \%$, amplification $=10$ ). $(B)$ The repulsive response as a function of concentration for amplification $=1,5,10$. Note the mirror symmetry between $B$ and Figure 3B.

logical implementation of these different forms for $P(\theta)$ is considered in the section 5, and their mathematical properties are analyzed below.

Although simulating the short-term response of axons in a steep gradient allows comparison with experiments using the pipette assay, more relevant to understanding the behavior of axons in vivo are simulations of the long-term response of axons to shallow gradients, as in 3D collagen gels (Lumsden \& Davies, 1983, 1986; Tessier-Lavigne et al., 1988; Colamarino \& Tessier-Lavigne, 1995; Keynes et al., 1997; Richards et al., 1997; VarelaEchavarria et al., 1997; Brose et al., 1999; Braisted et al., 2000; Caton et al., 2000; Patel et al., 2001; Nguyen Ba-Charvet et al., 2001; Shu \& Richards, 2001; Anderson et al., 2003). Figure 5 shows results of the model after 40 hours of simulated growth from an "explant," giving axons of length $800 \mu \mathrm{m}$. One hundred axons were initially distributed uniformly in a disk (explant) of radius $300 \mu \mathrm{m}$, with random initial directions. The disks drawn in Figure 5 are added simply to show the extent of the explant; as in the experimental case, it obscures the initial trajectories of the axons until they leave the explant. The trajectories are generated with the gradient increasing in the positive $y$ direction (upward in the figure: the actual gradient steepness present in standard 3D collagen gel experiments is unknown). All other parameters were as for the short trajectories above, with amplification $=10$. The explants generated by the model in this case resemble (at least qualitatively) explants seen experimentally (Lumsden \& Davies, 1983, 1986; Tessier-Lavigne et al., 1988; Colamarino \& Tessier-Lavigne, 1995; Keynes et al., 1997; Richards et al., 1997; Varela-Echavarria et al., 1997; Brose et al., 1999; Braisted et al., 2000; Caton et al., 2000; Patel et al., 2001; Nguyen Ba-Charvet et al., 2001; Shu \& Richards, 2001; Anderson et al., 2003). Figure 6A shows the mean $y$ displacement as a function of the concentration at the center of the explant. 


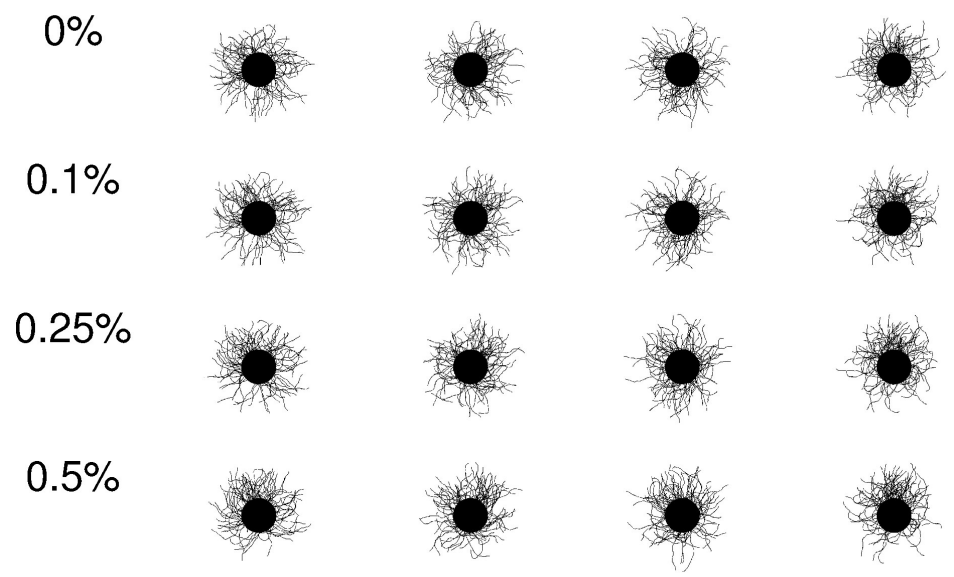

Figure 5: Long axon trajectories from an explant. Amplification $=10$, time simulated $=40$ hours. Gradient points upward, with the steepness indicated for each row. Each column represents a different random seed (the same within each column).

Again, sensitivity decays faster at high concentrations than low concentrations. Figure $6 \mathrm{~B}$ shows the analogous result in a repulsive gradient with the same parameters, using the $P(\theta) \propto(1-b(\theta))^{n}$ form. Again, sensitivity decays faster at low concentrations than high concentrations. Figure $6 \mathrm{C}$ shows mean $y$ displacement as a function of gradient steepness.

\section{Analysis of the Model}

Here we analyze certain aspects of the behavior of our model mathematically: the trajectory of the initial turn up the gradient and the concentration dependence of the sensitivity.

4.1 Calculation of Trajectories. The model is fundamentally stochastic: receptor binding is probabilistic, as is the position at which a new filopodium is generated (biased by the direction of maximum binding). However, for a simplified and deterministic version, a closed-form solution for the initial turn can be derived. Consider the "bare" (no filopodia) growth cone shown in Figure 7A, being attracted by an exponential gradient along the $x$ axis of form $C=C_{0} e^{\alpha x}$. (Refer to Table 2 for terminology.) The average binding $b(\theta)$ at each position on the growth cone is given by

$$
b(\theta)=\frac{C_{0} e^{\alpha(x+r \sin (\theta+\phi))}}{K_{D}+C_{0} e^{\alpha(x+r \sin (\theta+\phi))}} .
$$


A

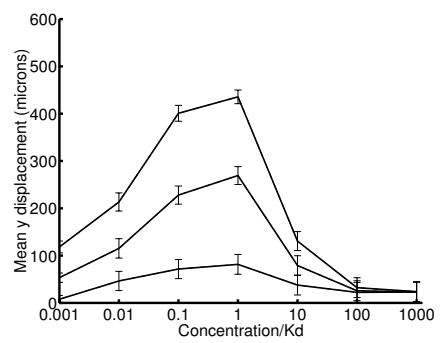

C

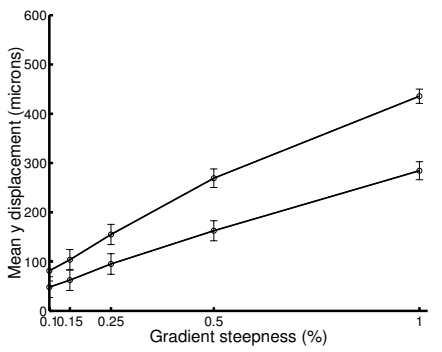

B

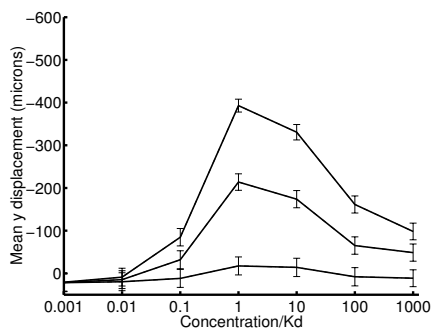

Figure 6: Analysis of long axon trajectories from an explant. Amplification = 10 , time simulated $=40$ hours. $(\mathrm{A}, \mathrm{B})$ Mean $y$ displacement as a function of concentration at the center of the explant $(\mathrm{A}=$ attraction, $\mathrm{B}=$ repulsion). The three curves in each case are (from top to bottom) for steepness $=1 \%, 0.5 \%$, and $0.1 \%$. (C) Mean $y$ displacement as a function of gradient steepness (top line, amplification $=10$; bottom line, amplification $=5$ ).

For shallow gradients, small $\alpha, b^{n}(\theta)$ is approximately

$$
b^{n}(\theta)=\left[\frac{\bar{C} e^{\alpha x}}{1+\bar{C} e^{\alpha x}}\right]^{n}\left[1+\frac{n \alpha r}{1+\bar{C} e^{\alpha x}} \sin (\theta+\phi)\right] .
$$

Define a normalized and amplified probability distribution:

$$
B(\theta)=\frac{b^{n}(\theta)}{\int_{-\pi / 2}^{+\pi / 2} b^{n}(\psi) d \psi} .
$$

Then

$$
\begin{aligned}
B(\theta) & =\frac{1+\frac{n \alpha r}{1+\bar{C} e^{\alpha x}} \sin (\theta+\phi)}{\pi+\frac{2 n \alpha r \sin \phi}{1+\bar{C} e^{\alpha x}}} \\
& \approx \frac{1}{\pi}\left[\left(1-\frac{2 n \alpha r \sin \phi}{\pi\left(\bar{C} e^{\alpha x}+1\right)}\right)+\frac{n \alpha r \sin (\theta+\phi)}{\bar{C} e^{\alpha x}+1}\right] .
\end{aligned}
$$


Table 2: Terminology for the Analysis of the Model.

\begin{tabular}{ll}
\hline Parameter & Definition \\
\hline$r$ & Growth cone radius \\
$\phi$ & Angle of growth cone relative to coordinate frame \\
$\theta$ & Angle over the growth cone surface $\epsilon[-\pi / 2, \pi / 2]$ \\
$\bar{C}$ & Ligand concentration normalized by $K_{D}($ dimensionless $)$ \\
$C_{0}$ & Ligand concentration at the origin \\
$\alpha$ & Gradient steepness, where concentration $=C_{0} e^{\alpha x}$ \\
$b(\theta)$ & Receptor binding as a function of $\theta$ \\
$B(\theta)$ & Normalized and amplified binding density \\
$n$ & Amplification parameter \\
$\eta$ & Time step in differential equation for $\phi$ \\
$\epsilon$ & Time step in differential equation for $x, y$ \\
$k$ & $\frac{2 \eta n \alpha r}{\pi(1+\bar{C})}$ \\
\hline
\end{tabular}

The binding-weighted direction $\langle\theta\rangle$ specified by this distribution is first to order in $\alpha$ and assuming $n \alpha$ is still small:

$$
\langle\theta\rangle=\int_{-\pi / 2}^{+\pi / 2} \psi B(\psi) d \psi \approx \frac{2 n \alpha r}{\pi\left(\bar{C} e^{\alpha x}+1\right)} \cos \phi .
$$

We now regard this as a small increment in the angle $\phi$ of the growth cone at each time step, that is, $\dot{\phi} \propto\langle\theta\rangle$. This leads to the set of coupled differential equations

$$
\dot{\phi}=\eta \frac{2 n \alpha r}{\pi\left(\bar{C} e^{\alpha x}+1\right)} \cos \phi, \quad \dot{x}=\epsilon \sin \phi, \quad \dot{y}=\epsilon \cos \phi,
$$

where $\eta$ and $\epsilon$ are small. These equations are analytically intractable. However, for this deterministic version of the model, it is reasonable to assume that the background concentration does not change significantly over the length of the initial turn. That is, we can assume $e^{\alpha x} \approx 1$ for the initial turn. The equation for $\dot{\phi}$ then becomes simply

$$
\dot{\phi}=\eta \frac{2 n \alpha r}{\pi(\bar{C}+1)} \cos \phi .
$$

Assuming $\phi(0)=0$, that is, the initial direction of the growth cone is perpendicular to the gradient as in our simulations, this has the solution

$$
\phi=\arcsin [\tanh (k t)],
$$

where $k=\frac{2 \eta n \alpha r}{\pi(1+\bar{C})}$. Solving the equations for $\dot{x}$ and $\dot{y}$ using this expression yields the following parametric trajectory:

$$
\begin{aligned}
x(t) & =\frac{\epsilon}{k} \ln (\cosh (k t)), \\
y(t) & =\frac{2 \epsilon}{k} \arctan (\tanh (k t / 2)) \cosh (k t) \sqrt{1-\tanh ^{2}(k t)} .
\end{aligned}
$$


A

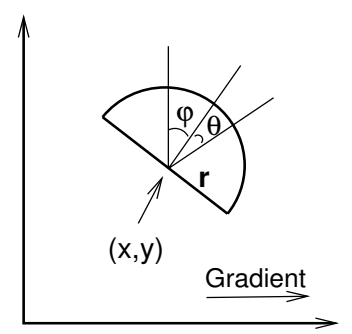

B

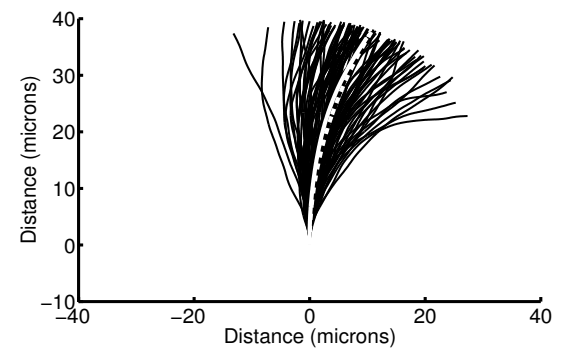

Figure 7: Analysis of the model. (A) Schematic of the terminology used in the analysis. (B) Comparison of simulated trajectories (black lines; mean trajectory is solid white line) with the prediction of the analysis (dashed white line).

Figure 7 compares this predicted trajectory for typical parameters with the actual trajectories produced by simulations of the full stochastic model. The solid white line in Figure 7B is the mean of the set of trajectories simulated with the full model, while the dashed white line is the trajectory generated by the closed-form solution above: there is a good match.

This analysis reveals that $k \propto \frac{n \alpha r}{1+\bar{C}}$ is a key determinant of the rate of turning. Any combination of $n, \alpha$, and $r$ that has the same product will have the same rate of turning. Thus, doubling the gradient steepness is equivalent to doubling the amplification, or doubling the width of the growth cone, and so on.

In the force version of the model, the total pull is the vector sum of the forces exerted by the filopodia. The total filopodial force can then be decomposed into a forwards (in the current direction of the growth cone) and turning (orthogonal to the current direction) force. The expected values of these forces are given by $\langle B(\theta) \cos (\theta)\rangle$ and $\langle B(\theta) \sin (\theta)\rangle$, respectively. For the turning force, we have

$$
\langle B(\theta) \sin (\theta)\rangle=\int_{-\pi / 2}^{\pi / 2} B(\psi) \sin (\psi) d \psi=\frac{n \alpha r}{2\left(\bar{C} e^{\alpha x}+1\right)} \cos \phi .
$$

This is exactly the same as the equivalent equation for the "angle" version of the model (see equation 4.1 ), except for the replacement of $2 / \pi$ by $1 / 2$. This demonstrates analytically that the force and angle models generate very similar trajectories.

4.2 Gradient Sensitivity. The concentration dependence of the gradient sensitivity can be understood from a first-order approximation of the concentration dependence of the filopodia initiation probability, $B(C)$, near 
the average concentration at the growth cone, $C_{0}$. By Taylor expansion,

$$
\begin{aligned}
B(C) & \approx B\left(C_{o}\right)+\left.\left(C-C_{o}\right) \frac{d B(C)}{d C}\right|_{C=C_{o}} \\
& =B\left(C_{o}\right)\left(1+\left.\frac{\left(C-C_{o}\right)}{C_{o}} \frac{C_{o}}{B\left(C_{o}\right)} \frac{d B(C)}{d C}\right|_{C=C_{o}}\right) .
\end{aligned}
$$

We have studied the concentration dependence of the sensitivity for gradients with constant fractional change across the growth cone, so the variation in the quantity $\frac{C-C_{0}}{C_{o}}$ across the growth cone is comparable at all concentrations. In addition, the normalization condition requires that $\int_{\theta_{\min }}^{\theta_{\max }} B\left(C_{o}\right)=1$, so the dependence on concentration and amplification of the effective gain $G_{e f f}$ is determined by $\left.G_{e f f} \equiv \frac{C_{o}}{B\left(C_{o}\right)} \frac{d B(C)}{d C}\right|_{C=C_{o}}$. By the chain rule,

$$
G_{e f f}=\left.\left.\frac{C_{o}}{B\left(C_{o}\right)} \frac{d B}{d b}\right|_{b\left(C_{o}\right)} \frac{d b}{d C}\right|_{C_{o}} .
$$

For brevity, we now drop the subscript from $C_{o}$. The last term in the expression above comes from the variation of the receptor binding with concentration and is independent of the form of the amplification:

$$
\frac{d b}{d C}=\frac{K_{d}}{\left(C+K_{d}\right)^{2}}=b^{2} \frac{K_{d}}{C^{2}} .
$$

Note that this function is approximately constant for $C \ll K_{d}$ and falls off rapidly when $C$ is larger than $K_{d}$, as a consequence of the receptor saturation. For the first form of amplification considered, $B(b)=A * b^{n}$, where $A$ is the normalization constant,

$$
G_{e f f}=\left(\frac{C}{A b^{n}}\right)\left(n A b^{(n-1)}\right)\left(b^{2} \frac{K_{d}}{C^{2}}\right)=\frac{n K_{d}}{C+K_{d}} .
$$

For $C \ll K_{d}, G_{e f f} \approx n$, while for $C \gg K_{d}, G_{e f f} \approx n K_{d} / C$. Thus, the amplification is effective at low concentrations, although the overall sensitivity drops, presumably because of statistical noise. At high concentrations, the amplification is ineffective because of the receptor saturation mentioned above.

For the first type of repulsion, $B(b)=A *(1-b)^{n}$, we find

$$
G_{e f f}=-n *\left(K_{d} / C\right) * \frac{b^{2}}{(1-b)}=-n \frac{C}{C+K_{d}} .
$$

This looks just like equation 4.2 , with $C / K_{d}$ substituted for $K_{d} / C$ and a change of sign. This relationship is a consequence of the fact that changing from $b$ to 
$1-b$ is formally equivalent to switching from amplifying bound receptors to amplifying unbound receptors.

Finally, for $B(b)=A *\left(1-b^{n}\right)$,

$$
G_{e f f}=-n *\left(K_{d} / C\right) * \frac{b^{(n+1)}}{\left(1-b^{n}\right)}=-n\left(\frac{K_{d}}{C+K_{d}}\right)\left(\frac{C^{n}}{\left(C+K_{d}\right)^{n}-C^{n}}\right) .
$$

For $C \ll K_{d}, G_{e f f} \approx-n\left(C / K_{d}\right)^{n}$, while for $C \gg K_{d}, G_{e f f} \approx-1$.

\section{Discussion}

Our model demonstrates that both the short-term response of axons to steep gradients and the long-term response of axons to shallow gradients can be reproduced from a set of relatively simple assumptions. The model makes specific and quantitative predictions for the amount and type of amplification of the binding signal required, the way the response varies with gradient steepness and with ligand concentration, how the turning in attractive gradients may differ from turning in repulsive gradients, and how intracellular signaling may vary between these two cases. The model also captures surprisingly well the degree of variability of axonal responses seen experimentally. While the majority of axons appear to be influenced by the gradient, some do not, even in the case of long trajectories. In experimental assays, a heterogeneous response is usually attributed to the presence of a heterogeneous population of axons. An alternative explanation suggested by our simulation data is that, at least in some cases, the stochastic nature of filopodium generation sometimes directs axons on an apparently unresponsive trajectory.

How might the sequence of events that leads to growth cone turning in the model be implemented biologically? As long as the products of the signaling cascade (Mueller, 1999; Song \& Poo, 2001; Yu \& Bargmann, 2001; Guan \& Rao, 2003) do not diffuse too rapidly, a shallow internal gradient (e.g., of G-protein signaling) will follow directly from the gradient in receptor binding. Amplification of this gradient can come from the cooperativity of receptor binding and from autocatalytic behavior, for instance, involving $\mathrm{Ca}^{2+}$ (Zheng, 2000; Gomez \& Spitzer, 2000; Hong et al., 2000; Gomez, Robles, Poo, \& Spitzer, 2001), and activator-inhibitor dynamics (Meinhardt, 1999; Song \& Poo, 2001). A gradient of activation of small GTPases of the Rho family (Hall, 1998), perhaps acting via Cdc42, could then produce a gradient of actin polymerization, with new filopodia more likely to sprout where the polymerization is enhanced. The positions of the filopodia are coupled to the direction of growth cone advance through adhesions between the filopodia and the substrate, and coupling between retrograde F-actin flow and microtubule extension (Suter \& Forscher, 2000; Zhou et al., 2002). Our model assumes that the overall rate at which new filopodia form is independent of the receptor binding (see Zheng, 2000). Since the outputs 
of the signaling cascade enhance or depress actin polymerization locally, some other process must be utilized to keep the overall level of polymerization constant. Such global effects could come from competition for limited resources for actin polymerization or from activator-inhibitor dynamics.

Experimental data suggest that the same pathways may be involved in both attractive and repulsive responses (Song \& Poo, 2001; Yu \& Bargmann, 2001). The most straightforward way to generate a repulsive response in our model is to effectively switch the roles of bound and unbound receptors by replacing $P(b) \propto b^{n}$ with $P(b) \propto(1-b)^{n}$. A possibly more realistic way of implementing this involves the silencing of an attractive response by heteromeric receptor complexes, such as the interaction of DCC and UNC-5 in netrin signaling (Yu \& Bargmann, 2001), where the attractive function of DCC is silenced by interaction with the UNC-5/netrin complex. If an attractive signaling complex (e.g., netrin/DCC) is saturated, the amount of intracellular signaling will decrease as the proportion of silencing receptorsligand complexes (UNC-5/netrin) increases. Alternatively, the products of the amplified cascade could depress rather than enhance polymerization activity. As shown in section 4.2, the chemotactic response is primarily determined by the first derivative of the amplification function, and thus the form $P(b) \propto b^{-n}$ produces a repulsive response approximately equal and opposite to the attractive response produced by $b^{n}$. This could arise from a $\mathrm{Ca}^{2+}$ set point (Gomez \& Spitzer, 2000; Petersen \& Cancela, 2000) for optimum axon outgrowth: the polymerization gradient is in the same direction as the $\mathrm{Ca}^{2+}$ gradient when the $\mathrm{Ca}^{2+}$ level is below the set point, and in the opposite direction when the level is above the setpoint (see Zheng, 2000; Hong et al., 2000).

Experimental measurements of the concentration dependence of attractive and repulsive chemotactic sensitivities should be able to distinguish between the different types of attractive and repulsive amplification considered here. This could be done with both the pipette assay and the collagen gel assay we have recently developed (Rosoff et al., 2004). Given the number of systems where the switch has been observed (Song \& Poo, 2001; Yu \& Bargmann, 2001; Guan \& Rao, 2003), it is quite possible that more than one type of behavior will be observed. We have chosen mathematical models for intracellular amplification that are relatively simple and realistic. As the signal transduction pathways become more fully elucidated, it will be possible to directly calculate from chemical kinetics the transformation between receptor binding and filopodia initiation probability. This transformation will undoubtedly depend on a variety of factors, but is likely to share the basic characteristics of one or more of the general forms we have considered here.

\section{Acknowledgments}

This work was funded by grants from the NIH, NSF, and Whitaker Foundation. 


\section{References}

Aletta, J. M., \& Greene, L. A. (1988). Growth cone configuration and advance: A time-lapse study using video-enhanced differential interference contrast microscopy. J. Neurosci., 8, 1425-1435.

Anderson, C. N., Ohta, K., Quick, M. M., Fleming, A., Keynes, R., \& Tannahill D. (2003). Molecular analysis of axon repulsion by the notochord. Development, 130, 1123-1133.

Argiro, V., Bunge, M. B., \& Johnson, M. I. (1985). A quantitative study of growth cone filopodial extension. Journal of Neuroscience Research, 13, 149-162.

Barkai, N., \& Leibler, S. (1997). Robustness in simple biochemical networks. Nature, 387, 913-917.

Bentley, D., \& Toroian-Raymond A. (1986). Disoriented pathfinding by pioneer neuron growth cones deprived of filopodia by cytochalasin treatement. $\mathrm{Na}-$ ture, 323, 712-715.

Berg, H. C., \& Purcell, E. M. (1977). Physics of chemoreception. Biophysical Journal, 20, 193-219.

Braisted, J. E., Catalano, S. M., Stimac, R. Kennedy T. E., Tessier-Lavigne, M., Shatz, C. J., \& O'Leary, D. D. M. (2000). Netrin-1 promotes thalamic axon growth and is required for proper development of the thalamocortical projection. J. Neurosci., 20, 5792-5801.

Bray, D. B., \& Chapman, K. (1985). Analysis of microspike movements on the neuronal growth cone. J. Neurosci., 5, 3204-3213.

Brose, K., Bland, K. S., Wang, K. H., Arnott, D., Henzel, W., Goodman, C. S., Tessier-Lavigne, M., \& Kidd, T. (1999). Slit proteins bind robo receptors and have an evolutionarily conserved role in repulsive axon guidance. Cell, 96, 795-806.

Buettner, H. M. (1995). Computer simulation of nerve growth cone filopodial dynamics for visualization and analysis. Cell Motil. Cytoskeleton, 32, 187-204.

Caton, A., Hacker, A., Naeem, A., Livet, J., Maina, F., Bladt, F., Klein, R., Birchmeier, C., \& Guthrie, S. (2000). The branchial arches and HGF are growthpromoting and chemoattractant for cranial motor axons. Development, 127, 1751-1766.

Charron, F., Stein, E., Jeong, J., McMahon, A. P., \& Tessier-Lavigne, M. (2003). The morphogen sonic hedgehog is an axonal chemoattractant that collaborates with netrin-1 in midline axon guidance. Cell, 113, 11-23.

Chien, C. B., Rosenthal, D. E., Harris, W. A., \& Holt, C.E. (1993). Navigational errors made by growth cones without filopodia in the embryonic Xenopus brain. Neuron, 11, 237-251.

Colamarino, S. A., \& Tessier-Lavigne, M. (1995). The axonal chemoattractant netrin-1 is also a chemorepellent for trochlear motor axons. Cell, 81, 621-629.

Davenport, R. W., Dou. P., Rehder, V., \& Kater, S. B. (1993). A sensory role for neuronal growth cone filopodia. Nature, 361, 721-724.

de la Torre, J. R., Höpker, V. H., Ming, G-l., \& Poo, M-M. (1997). Turning of retinal growth cones in a netrin-1 gradient mediated by the netrin receptor DCC. Neuron, 19, 1211-1224. 
Devreotes, P. N., \& Zigmond, S. H. (1988). Chemotaxis in eukaryotic cells: A focus on leukocytes and Dictyostelium. Ann. Rev. Cell. Biol., 4, 649-686.

Dickson, B. J. (2002). Molecular mechanisms of axon guidance. Science, 298, 1959-1964.

Eisenbach, M. (1996). Control of bacterial chemotaxis. Molecular Microbiology, 20, 903-910.

Goldberg, D. J., \& Burmeister, D. W. (1986). Stages in axon formation: Observations of growth of Aplysia axons in culture using video-enhanced contrastdifferential interference contrast microscopy. J. Cell. Biol., 103, 1921-1931.

Gomez, T. M., Robles, E., Poo, M-M., \& Spitzer, N. C. (2001). Filopodial calcium transients promote substrate-dependent growth cone turning. Science, 291, 1983-1987.

Gomez, T. M., \& Spitzer, N. C. (2000). Regulation of growth cone behavior by calcium: New dynamics to earlier perspectives. J. Neurobiol., 44, 174-183.

Goodhill, G. J. (1997). Diffusion in axon guidance. European Journal of Neuroscience, 9, 1414-1421.

Goodhill, G. J. (1998). Mathematical guidance for axons. Trends in Neurosciences, 21, 226-231.

Goodhill, G. J., \& Urbach, J. S. (1999). Theoretical analysis of gradient detection by growth cones. Journal of Neurobiology, 41, 230-241.

Gordon-Weeks, P. R. (2000). Neuronal growth cones. Cambridge: Cambridge University Press.

Guan, K. L., \&, Rao Y. (2003). Signalling mechanisms mediating neuronal responses to guidance cues. Nature Rev. Neurosci., 4, 941-956.

Gundersen, R. W., \& Barrett, J. N. (1979). Neuronal chemotaxis: Chick dorsalroot axons turn toward high concentrations of nerve growth factor. Science, 206, 1079-1080.

Hall, A. (1998). Rho GTPases and the actin cytoskeleton. Science, 279, 509-514.

Haykin, S. (1999). Neural networks: A comprehensive foundation. Upper Saddle River, NJ: Prentice Hall.

Hely, T. A., \& Willshaw, D. J. (1998). Short term interactions between microtubules and actin filaments underlie long term behaviour in neuronal growth cones. Proc. Roy. Soc. B, 265, 1801-1807.

Hong, K., Hinck, L., Nishiyama, M., Poo, M-M., \& Tessier-Lavigne, M. (1999). A ligand-gated association between cytoplasmic domains of UNC5 and DCC family receptors converts netrin-induced growth cone attraction to repulsion. Cell, 92, 205-215.

Hong, K., Nishiyama, M., Henley, J., Tessier-Lavigne, M., \& Poo, M-M. (2000). Calcium signalling in the guidance of nerve growth by netrin-1. Nature, 403, 93-98.

Höpker, V. H., Shewan, D., Tessier-Lavigne, M., Poo, M-M., \& Holt, C. (1999). Growth-cone attraction to netrin-1 is converted to repulsion by laminin-1. Nature, 401, 69-73.

Huber, A. B., Kolodkin, A. L., Ginty, D. D., \& Cloutier, J. F. (2003). Signaling at the growth cone: Ligand-receptor complexes and the control of axon growth and guidance. Annu. Rev. Neurosci., 26, 509-563.

Hutson, L. D., \& Chien, C.-B. (2002). Pathfinding and error correction by retinal axons: The role of astray/robo2. Neuron, 33, 205-217. 
Keynes, R., Tannahill, D., Morgenstern, D. A., Johnson, A. R., Cook, G. M. W., \& Pini, A. (1997). Surround repulsion of spinal sensory axons in higher vertebrate embryos. Neuron, 18, 889-897.

Kim, M. D., Kolodziej, P., \& Chiba, A. (2002). Growth cone pathfinding and filopodial dynamics are mediated separately by Cdc42 activation. J. Neurosci., 22, 1794-1806.

Lumsden, A. G. S., \& Davies, A. M. (1983). Earliest sensory nerve fibres are guided to peripheral targets by attractants other than nerve growth factor. Nature, 306, 786-788.

Lumsden, A. G. S. and Davies, A. M. (1986). Chemotropic effect of specific target epithelium in the developing mammalian nervous system. Nature, 323, 538539.

Meinhardt, H. (1999). Orientation of chemotactic cells and growth cones: Models and mechanisms. Journal of Cell Science, 112, 2867-2874.

Ming, G., Henley, J., Tessier-Lavigne, M., Song, H., \& Poo, M. (2001). Electrical activity modulates growth cone guidance by diffusible factors. Neuron, 29, 441-452.

Ming, G-I., Song, H-J., Berninger, B., Holt, C. E., Tessier-Lavigne, M., \& Poo, M-M. (1997). cAMP-dependent growth cone guidance by netrin-1. Neuron, $19,1225-1235$.

Ming, G., Wong, S. T., Henley, J., Yuan, X., Song, H., Spitzer, N., \& Poo, M. (2002). Adaptation in the chemotactic guidance of nerve growth cones. Nature, 417, 411-418.

Moghe, P. V., \& Tranquillo, R. T. (1995). Stochasticity in membrane-localized ligand-receptor G-protein binding-consequences for leukocyte movement behavior. Ann. Biomed. Eng., 23, 257-267.

Mueller, B. K. (1999). Growth cone guidance: First steps towards a deeper understanding. Annu. Rev. Neurosci., 22, 351-388.

Myers, P. Z., \& Bastiani, M. J. (1993). Growth cone dynamics during the migration of an identified commissural growth cone. J. Neurosci., 13, 127-143.

Nguyen Ba-Charvet, K. T., Brose, K., Ma, L., Wang, K. H., Marillat, V., Sotelo, C., Tessier-Lavigne, M., \& Chedotal, A. (2001). Diversity and specificity of actions of Slit2 proteolytic fragments in axon guidance. J. Neurosci., 21, 42814289.

Nishiyama, M., Hoshino, A., Tsai, L., Henley, J. R., Goshima, Y., Tessier-Lavigne, M., Poo, M. M., \& Hong K. (2003). Cyclic AMP/GMP-dependent modulation of $\mathrm{Ca} 2+$ channels sets the polarity of nerve growth-cone turning. Nature, 424. 990-995.

O'Connor, T. P., Duerr, J. S., \& Bentley, D. (1990). Pioneer growth cone steering decisions mediated by single filopodial contacts in situ. J. Neurosci., 10, 39353946.

Parent, C. A., \& Devreotes, P. N. (1999). A cell's sense of direction. Science, 284, 765-770.

Patel, K., Nash, J. A., Itoh, A., Liu, Z., Sundaresan, V., \& Pini, A. (2001). Slit proteins are not dominant chemorepellents for olfactory tract and spinal motor axons. Development, 128, 5031-5037.

Petersen, O. H., \& Cancela, J. M. (2000). Attraction or repulsion by local Ca(2+) signals. Curr. Biol., 10, R311-314. 
Rehder, V., \& Kater, S. B. (1996). Filopodia on neuronal growth cones: Multifunctional structures with sensory and motor capabilities. Seminars in the Neurosciences, 8, 81-88.

Richards, L. J., Koester, S. E., Tuttle, R., \& O'Leary, D. D. M. (1997). Directed growth of early cortical axons is influenced by a chemoattractant released from an intermediate target. J. Neurosci., 17, 2445-2458.

Robert, M. E., \& Sweeney, J. D. (1997). Computer model: Investigating role of filopodia-based steering in experimental neurite galvanotropism. J. Theor. Biol., 188, 277-288.

Rosoff, W. J., Urbach, J. S., Esrick, M., McAllister, R. G., Richards, L. J., \& Goodhill, G. J. (2004). A novel chemotaxis assay reveals the extreme sensitivity of axons to molecular gradients. Nat. Neurosci., 7, 678-682.

Shu, T., \& Richards, L. J. (2001) Cortical axon guidance by the glial wedge during the development of the corpus callosum. J. Neurosci., 21, 2749-2758.

Song, H., Ming, G., He, Z., Lehmann, M., Tessier-Lavigne, M., \& Poo, M-M. (1998). Conversion of neuronal growth cone responses from repulsion to attraction by cyclic nucleotides. Science, 281, 1515-1518.

Song, H. J., Ming, G. L., \& Poo, M-M. (1997). cAMP-induced switching in turning direction of nerve growth cones Nature, 388, 275-279.

Song, H., \& Poo, M-M. (2001). The cell biology of neuronal navigation. Nat. Cell. Biol., 3, E81-88.

Steketee, M., Balazovich, K., \& Tosney, K. W. (2001). Filopodial initiation and a novel filament-organizing center, the focal ring. Mol. Biol. Cell., 12, 2378-2395.

Steketee, M. B., \& Tosney, K. W. (1999). Contact with isolated sclerotome cells steers sensory growth cones by altering distinct elements of extension. $J$. Neurosci., 19, 3495-3506.

Suter, D. M., \& Forscher, P. (2000). Substrate-cytoskeletal coupling as a mechanism for the regulation of growth cone motility and guidance. J. Neurobiol., $44,97-113$.

Tanaka, E., \& Sabry, J. (1995). Making the connection: Cytoskeletal rearrangements during growth cone guidance. Cell, 83, 171-176.

Tessier-Lavigne, M., Placzek, M., Lumsden, A. G. S., Dodd, J., \& Jessell, T. M. (1988). Chemotropic guidance of developing axons in the mammalian central nervous system. Nature, 336, 775-778.

Tranquillo, R. T. (1990). Models of chemical gradient sensing by cells. In W. Alt \& G. Hoffman (Eds.), Biological motion, New York: Springer-Verlag. (pp. 415441).

Tranquillo, R. T., \& Lauffenburger, D. A. (1987). Stochastic-model of leukocyte chemosensory movement. J. Math. Bio., 25, 229-262.

Varela-Echavarria, A., Tucker, A., Püschel, A. W., \& Guthrie, S. (1997). Motor axon subpopulations respond differentially to the chemorepellents netrin-1 and semaphorin D. Neuron, 18, 193-207.

Wang, F. S., Liu, C. W., Diefenbach, T. J., \& Jay, D. G. (2003). Modeling the role of myosin 1c in neuronal growth cone turning. Biophys. J., 85, 3319-3328.

Yu, T. W., \& Bargmann, C. I. (2001). Dynamic regulation of axon guidance. Nat. Neurosci., 4 (Suppl). 1169-1176.

Zheng, J.Q. (2000). Turning of nerve growth cones induced by localized increases in intracellular calcium ions. Nature, 403, 89-93. 
Zheng, J. Q., Felder, M., Conner, J. A., \& Poo, M-M. (1994). Turning of growth cones induced by neurotransmitters. Nature, 368, 140-144.

Zheng, J. Q., Wan, J-J., \& Poo, M-M. (1996). Essential role of filopodia in chemotropic turning of nerve growth cone induced by a glutamate gradient. J. Neurosci., 16, 1140-1149.

Zhou, F. Q., Waterman-Storer, C. M., \& Cohan, C. S. (2002). Focal loss of actin bundles causes microtubule redistribution and growth cone turning. J. Cell Biol., 157, 839-849.

Zigmond, S. H. (1977). Ability of polymorphonuclear leukocytes to orient in gradients of chemotactic factors. J. Cell. Biol., 75, 606-616.

Received February 4, 2004; accepted April 29, 2004. 